\title{
Erratum to: Long-Term In Vivo Imaging of Luciferase-Based Reporter Gene Expression in Arabidopsis Roots
}

\section{Wei Xuan, Davy Opdenacker, Steffen Vanneste, and Tom Beeckman}

\section{Erratum to:}

Chapter 13 in: Daniela Ristova and Elke Barbez (eds.),

Root Development: Methods and Protocols,

Methods in Molecular Biology, vol. 1761,

https://doi.org/10.1007/978-1-4939-7747-5_13

The original version of Chapter 13 was inadvertently published with incorrect device information, this has now been updated. 